\title{
Dissociating meat from its animal origins: A systematic literature review
}

\author{
Nora C.G. Benningstad, Jonas R. Kunst* \\ Department of Psychology, University of Oslo, Norway
}

\section{A R T I C L E I N F O}

\section{Keywords:}

Animals

Cognitive dissonance

Dissociation

Meat consumption

Meat paradox

Literature review

\begin{abstract}
A B S T R A C T
Meat eaters often have an ambivalent relationship with the common practice of killing animals for food. They enjoy the taste of meat but dislike the harming of animals that it entails. This moral conflict, often referred to as the 'meat paradox,' tends to result in cognitive dissonance that meat eaters need to resolve. One of the arguably most basic strategies to deal with this dissonance is to cognitively dissociate meat from its animal origins. Whereas philosophers for long time have theorized about the role of such dissociation for consumer behavior, researchers have only recently started to empirically investigate the phenomenon. Here, we present the first systematic literature review of research on consumers' tendency to dissociate meat from its animal origins. Twenty-one publications comprising eight qualitative, one mixed-methods, four correlational, and twenty experimental/interventional studies were identified, which all provided support for the central psychological role of dissociation for meat consumption. However, the review also revealed the need for further research on moderating variables such as gender, age and generation, dietary styles, and people's place of living, including cross-cultural differences. Strikingly, no study so far seems to have included behavioral outcomes, urging the need for future research on how dissociation might affect behavior.
\end{abstract}

\section{Introduction}

Ever since pre-historic times, meat has been a central part of people's diet in most cultures (Leroy \& Praet, 2015). During the 20th century, there was a pronounced rise in global meat consumption, mainly caused by economic growth, urbanization and developments in meat production technology (Delgado, 2003; Graça, Calheiros, \& Oliveira, 2014). Despite the increasingly important role of meat for people's diets, meat eaters often have an ambivalent relationship with killing animals for food. Most people enjoy the taste of meat but dislike the harming of animals that meat production inevitably involves. This phenomenon has been referred to as the "meat paradox," and argued to result in a cognitive dissonance amongst meat eaters (Loughnan, Haslam, \& Bastian, 2010). Several strategies that people use to deal with this dissonance have been identified. These strategies can be direct and explicit such as denial of animals' pain, moral status or intelligence, endorsement of a hierarchy in which humans are placed above nonhuman animals, embracement of a pro-meat attitude, and justifications of meat consumptions based on nutritional or normative grounds (see, e.g., Bastian, Loughnan, Haslam, \& Radke, 2012; Piazza et al., 2015; Rothgerber, 2013). However, they can also rely on processes that render such strategies and justifications unnecessary. One such process is the dissociation of meat from its animal origins (Kunst \& Hohle, 2016;
Rothgerber, 2013; Tian, Hilton, \& Becker, 2016).

The dissociation process has for a long time been a subject in philosophy as well as public discourse, and many have argued that mentally separating meat from its animal source has profound effects on meat consumption (Foer, 2009; Joy, 2011; Singer, 1995). However, this dissociation hypothesis has only relatively recently been tested empirically and, although it has garnered attention from nutritional science, sociology, marketing and psychology, there has to date been no comprehensive synthesis of this research literature. The purpose of the present systematic review was therefore to provide an overview of the state of evidence on the dissociation hypothesis and its moderating factors.

\subsection{Dissociation as a mean to prevent and reduce cognitive dissonance}

At the core of the meat paradox is the experience of cognitive dissonance. Cognitive dissonance theory proposes that situations involving conflicting behaviors, beliefs or attitudes produce a state of mental discomfort (Festinger, 1957). Two cognitions are said to stand in a dissonant or conflictual relationship when one psychologically does not fit the other, as is the case when a person eats meat yet does not want to harm animals. Moreover, it has been argued that one qualifying condition for dissonance to emerge is that individuals feel responsible for

\footnotetext{
* Corresponding author. Department of Psychology, University of Oslo, Postboks 1094, Blindern, 0317, Oslo, Norway.

E-mail address: j.r.kunst@psykologi.uio.no (J.R. Kunst).
} 
their actions (Cooper \& Fazio, 1984). Cognitive dissonance theory further centers on the idea that people strive for consistency between their cognitions, and that they apply a variety of methods to achieve it. Consistency between cognitions and actions is most common, but it is easy to find examples of inconsistent cognitions (Festinger, 1957). Importantly, a person holding dissonant cognitions only rarely experiences them psychologically as inconsistent in the long term. Instead, more or less successful attempts are made to explain or rationalize them, thereby reducing dissonance.

Common methods to (re)achieve consistency between one's attitudes and/or behavior include changing or justifying one or both of them, adding new parts to the cognition, or entirely distorting one's perception and information about the world (Festinger, 1962). Eating meat does not psychologically fit together with not wanting to hurt animals, and together they cause an internal conflict. Moreover, most individuals would be expected to feel some degree of agency when it comes to their food choices. As mentioned, there are many dissonancereducing strategies that omnivores can employ to reduce the discomfort that arises. However, one way to prevent cognitive dissonance from emerging in the first place is to simply dissociate meat from its animal origins.

In earlier hunter-gatherer and agricultural societies, people were quite familiar with the animals they consumed. It can, hence, be argued that they had a clear association between meat and animals. During the mid-19th century, a combination of factors, such as industrialization, urbanization, population growth and increased purchasing power opened up for large-scale, intensive raising and slaughtering of animals for meat production purposes (Segers, 2012). Consequently, an increasing number of people became largely separated from animals used in food production and, thus, had less contact with the animals they consumed.

Singer (1995) argued that obtaining meat from stores or restaurants is the culmination of a long process, of which all but the finished product is concealed. In fact, in most industrialized societies, the majority of consumers only rarely take part in the preparation or processing of meat that is needed before it can be consumed. This processing often includes beheading, removal of entrails, flaying, or plucking and cutting the meat into pieces. It, hence, involves the removal of the typical animal characteristics from the meat, and the consumer is left with neatly packed, ready-to-cook pieces with none, or very few reminders of the animal. This process gives people little reason to associate what they are buying and eating with a once living and breathing animal. It has even been argued that most people living in contemporary industrialized societies are so far removed from the animals used in food production that the most meaningful and frequent action they take towards these animals involves the consumption of their flesh (Rothgerber \& Mican, 2014; Singer, 1995).

Dissociation can also be observed at a linguistic level, demonstrating its cultural entrenchment. The language commonly used to refer to meat products often renders animals absent from the consumer's consciousness and conceals its animal origins (Adams, 1990; Singer, 1995). For instance, in English, dead animals prepared as food are referred to with terms originally introduced by French-speaking Normans such as pork (not pig), beef (not cow), and veal (not calf; Morton, 2004), and one could argue that they nowadays function as euphemism. Additionally, words like burger, sausage and bacon can make the animal origins even more distant. In fact, the term "meat" itself can be argued to be part of the concealing of these animal origins. It was originally not reserved for animal meat but could be used to describe any type of solid food (Singer, 1995).

Although dissociation often takes place implicitly (i.e., outside of conscious awareness) and thereby prevents feelings of discomfort to emerge in the first place, it can also be an explicit strategy that people use to actively manage emotional responses resulting from the meat paradox. For instance, to reduce their discomfort, people can try not to connect meat to animals (Graça et al., 2014; Rothgerber, 2013) and avoid situations that may disrupt this dissociation (Holm, 2018; Schröder \& McEachern, 2004).

This definition of meat-animal dissociation shares similarities with, and is facilitated by, other constructs and processes. Bastian and Loughnan (2017) convincingly argued that people rarely consciously reflect on the moral implications of eating meat because societal mechanisms keep people's cognitive dissonance in place. Two such mechanisms are ritualization and institutionalization. People often partake in rituals and traditions without reflecting on their rationale or consequences. Hence, rituals help by embedding behavior, which people otherwise would have had conflicting feelings about, in social norms and institutions. For instance, the marketing, sale and production of meat in ways that conceal its animal origins can be seen as an institutionalization that facilitates the dissociation process (Bastian \& Loughnan, 2017; Singer, 1995), preventing dissonance to emerge and thereby reducing the need to use dissociation as an active coping strategy.

Dissociation can also be understood in light of how people generally deal with disgust-eliciting objects as outlined by Rozin (2008). Most closely related to the dissociation process is people's tendency to directly suppress the disgust implications of an object. This tendency converges with the explicit use of dissociation as a strategy, which suppresses knowledge of meat's disgust-eliciting animal source. Second, dissociation is related to the principle of hedonic adaptation. Such adaptation involves that an initially disgusting object loses its potency to elicit an emotional response the more people are exposed to it. In line with this process, individuals who are familiar with meat production and processing tend to experience less discomfort when meat is associated with animal origins (Kunst \& Haugestad, 2018).

\subsection{Research objectives}

Whereas many consumers' ambivalent relationship to eating meat has been a topic of scholarly debates for a longer period of time, researchers have only recently started to empirically investigate dissociation as an underlying psychological process bolstering meat consumption. The aim of this review was therefore to systematically synthesize the current state of evidence on the dissociation hypothesis. Furthermore, based on the review, a second aim was to identify unresolved questions, limitations and avenues for future research.

\section{Method}

A comprehensive search was conducted following three consecutive steps. First, a search of the electronic databases Google Scholar, ISI Web of Science and PsycINFO was carried out. Studies were identified by using combinations of the theoretical search terms "dissociation," "meat paradox," "cognitive dissonance," "conflicted omnivore*," and "meat-animal link," paired with contextual terms such as "meat," "meat consumption," "meat eat"," "diet," "meat avoidance," "meat-based," and "plant-based." This initial search yielded a large number of studies ( $>2000$ ). To be included in the review, studies from this pool had to focus on how participants connected, or disconnected, meat from its animal origins. The primary investigator determined this by manually inspecting the articles' titles, and, if relevant, abstract and main text, and this selection was further validated by a second researcher.

Next, studies that cited the articles obtained in the first step were identified using Google Scholar, and included if they matched the inclusion criteria. Third, the reference sections of the articles retained in the first two steps were searched in order to identify additional articles that met the inclusion criteria. A number of articles included in this review consisted of several studies, of which only some focused on dissociation. In such cases, only the relevant studies were considered. In each step, the search included all publication dates. The review was performed throughout March 2019, with three additional studies being added in response to the review process in September 2019. 


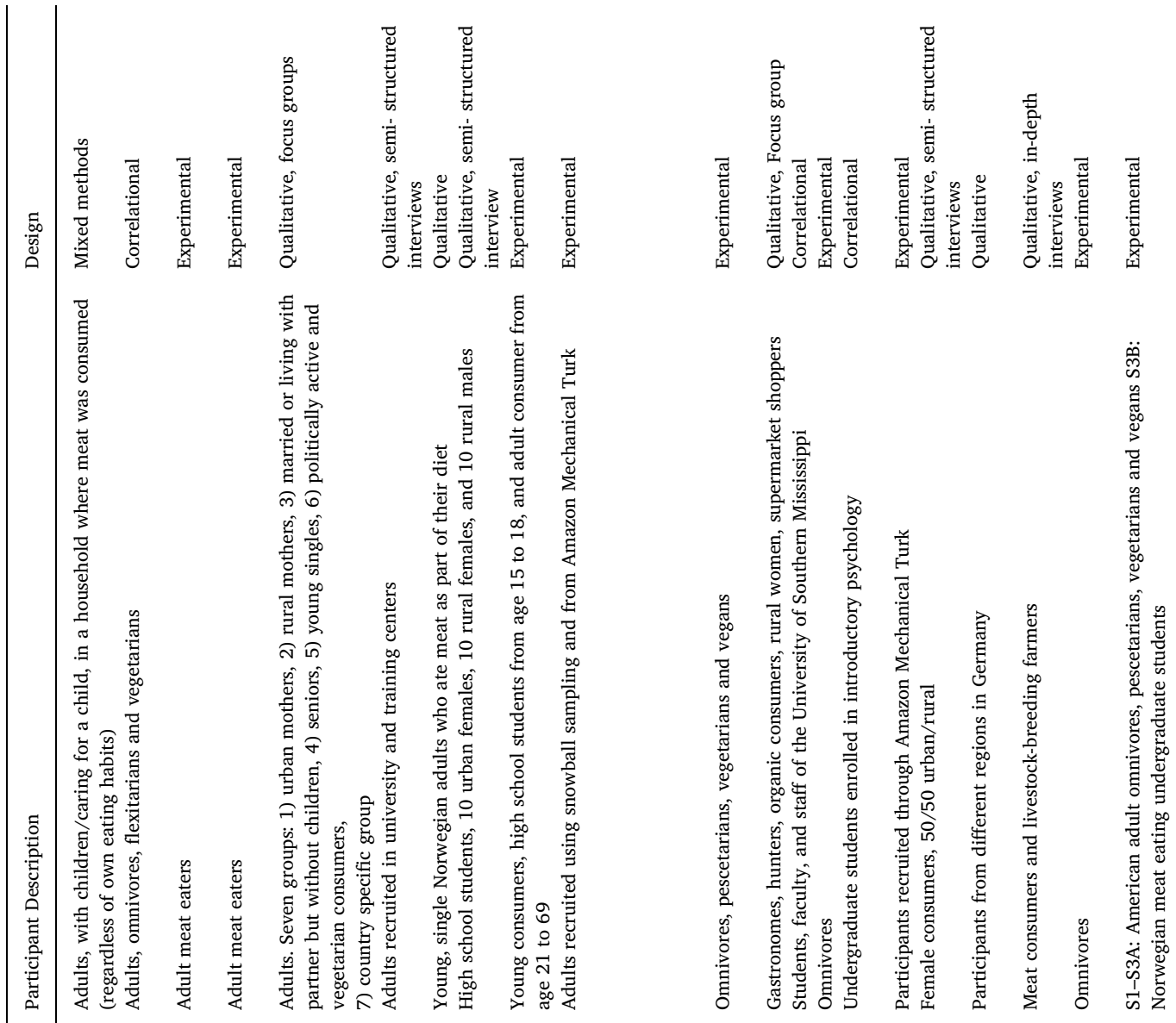

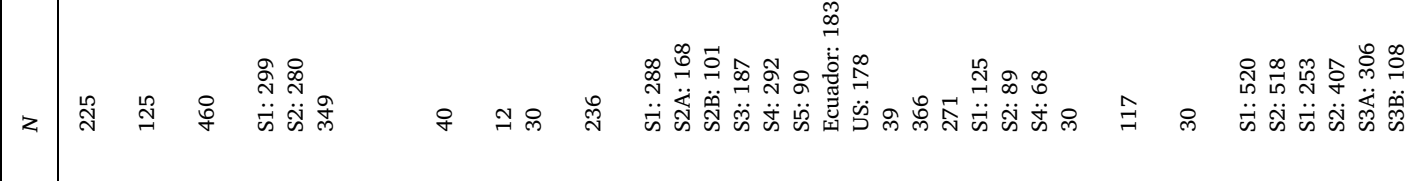

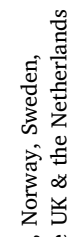

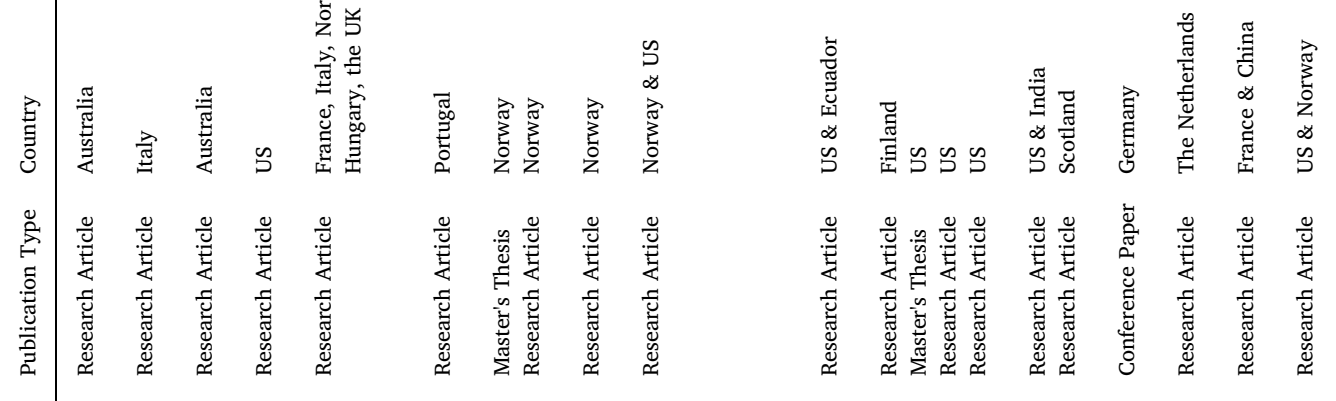




\section{Results}

Following the procedure outlined above, a total of 33 studies presented in 21 scientific publications were identified that met the inclusion criteria. Eight of these studies were qualitative, one used mixedmethods, four were correlational, and twenty experimental. Table 1 lists all studies, including publication type, country, number and description of participants, as well as the type of design (i.e., qualitative, correlational, experimental or mixed methods). Please note that we use the term 'experimental' to refer to any type of study that used random assignment to different conditions to manipulate a variable and test for causal effects. This definition, hence, includes studies that test interventions and excludes correlational studies. As the overview in Table 1 demonstrates, research on dissociation has increased especially in recent years, with 24 of the 33 studies appearing after 2015. Moreover, research using quantitative methods (correlational and experimental) has increased in particular since 2015.

\section{Evidence for the dissociation process}

Qualitative, correlational and experimental studies generally converge in support of the dissociation hypothesis, suggesting that (a) cues that more or less implicitly interrupt or facilitate dissociation influence the dissonance that meat consumers experience, and (b) that meat eaters actively employ dissociation as a coping strategy to regulate this experience of dissonance.

Evidence for the role of implicit dissociation processes comes from several studies. Evans and Miele (2012), who investigated consumers' interactions with animal food products, showed that the fast pace of food shopping, the presentation of animal foods (e.g., highly processed foods), and everyday food vocabulary (e.g., pork, beef and mutton) reduced consumers' ability to reflect upon the animal origins of the food they bought. By contrast, Holm (2018) identified factors that triggered the connection between meat and animals, thereby disrupting the dissociation process. For instance, cheap meat products, observing transportation of animals to the slaughterhouse, and a low degree of processing were reported as potent triggers of the animal-meat connection. Yet, as the latter research was published in form of a master's thesis, its results have not been peer-reviewed and, thus, should be interpreted with caution.

Additional studies provide insights into how consumers often have difficulties making meat-animal connections. Kubberød et al. (2002) found that participants had difficulties verbalizing the animal origins of different meat products, suggesting that dissociation was deeply entrenched in their consuming habits. Simons et al. (2018) found that there was no agreement about what should be considered as meat. Whereas unprocessed meat such as steak was consistently perceived as meat, more processed meat was sometimes not seen as meat at all and was often not considered when the informants reported the frequency of their meat eating.

Taking these qualitative findings to the lab, Kunst and Hohle (2016) experimentally demonstrated how daily processes of presenting, preparing and referring to meat influence dissociation. The authors showed that processing of meat and removing its animal characteristics (e.g., the head of an animal roast) facilitated dissociation, thereby leading to less disgust and empathy for the killed animal and higher intentions to eat meat. By contrast, making the animal-meat link salient by displaying a living animal in a meat dish advertisement or replacing euphemisms in a menu (e.g., "beef" with "cow") reduced dissociation. Importantly, the authors also showed that interrupting dissociation led to more willingness to choose a vegetarian dish, which may be relevant for interventions aiming to reduce meat consumption in applied settings. Kunst and Hohle's (2016) paradigm was successfully replicated and extended by Earle et al. (2019). The latter authors showed that distress about one's meat consumption (arguably functioning as a proxy measure of one's experienced dissonance) in addition to the disgust and empathy response identified by Kunst and Hohle (2016) explained the negative effects of interrupting dissociation on intentions to consume meat.

Finally, Kunst and Hohle (2016) showed that people scoring high on the dissociation dimension of the Meat Eating Justification scale were most affected by cues priming the animal-meat connection. Rothgerber, a pioneer in the study of dissociation, developed this scale as part of his 2013 study (Rothgerber, 2013). Questions assessing dissociation include whether individuals try not to think about the animal origins of meat. Kunst and Hohle's (2016) findings hence suggest that people who experience the most discomfort when reminded about meat's animal origins, are those who most actively engage in dissociation in the first place.

Additional insights into the potential mediators of the dissociation process were provided by Zickfeld et al. (2018) and Piazza et al. (2018). Both groups of authors tested whether perceptions of cuteness - classically linked to caring responses - would explain why disrupting the dissociation process by showing animals reduces people's willingness to eat meat. Whereas Zickfeld et al. (2018) found that empathy outperformed cuteness perceptions as a mediator of the dissociation process, Piazza et al. (2018) found that appetite for a meat dish was reduced when the dish was paired with a (cute) baby animal but not when it was paired with an adult animal.

Several studies demonstrate consumers' active use of dissociation as a strategy to cope with cognitive dissonance and resulting discomfort. Graça et al. (2014) drew on moral disengagement theory (Bandura, 1999) to explore consumers' use of moral disengagement when considering the impacts of their food habits. Moral disengagement has been argued to allow individuals to maintain self-serving behaviors that are harmful to others through disengaging self-regulatory mechanisms that govern their moral conduct. The authors found that dissociation emerged as an additional moral disengagement strategy that was not part of Bandura's original framework. The participants reported that one central strategy they used when consuming meat was to try not to think of the lives and deaths of the animals they ate. Similarly, Schröder and McEachern (2004) found that it was common among informants to suppress thoughts about animal production and animal slaughter. In line with this, Simons et al. (2018) found that many of their informants separated issues related to animal husbandry from their thoughts about meat consumption to be able to continue to enjoy eating meat. However, it is important to note that the latter study was presented as a conference paper and is therefore not peer-reviewed to the same extent as a journal article would be. Some studies also found that people prefer or actively choose to buy and eat meat that does not remind them of the animal origins (Holm, 2018; Te Velde et al., 2002). Similarly, Te Velde et al. (2002) found that meat consumers and farmers use strategies such as concealment and detachment to be able to eat and rear livestock.

While providing general support for the role of dissociation for consumer behavior, the reviewed studies also identified factors that potentially influence the process of dissociation and the need to dissociate. The most central moderators of dissociation were gender, age, place of living, culture and dietary patterns, which will be discussed in the following sections.

\subsection{Gender differences}

A common question in research on attitudes towards meat involves whether gender differences exist (Leroy \& Praet, 2015; Rosenfeld, 2018; Rothgerber, 2013; Ruby, 2012). As such differences are often observed for meat consumption (Rosenfeld, 2018) and concern for animal welfare (Rothgerber, 2013), gender may also play a role for dissociation processes. Several of the studies included in this literature review investigated whether gender might affect how much dissociation is relied upon as a dissonance-reducing strategy and how consumers react when dissociation is made difficult.

In Kubberød and colleagues' (2002) qualitative study on disgust and 
meat consumption, substantial differences emerged between the twenty young female and ten young male informants. Men were more aware of the origins of different types of meat, yet reported not reflecting on these origins when consuming it. Women to larger extent reported that they did not want to associate the meat they ate with a living animal, and that reminders would make them uncomfortable and sometimes even unable to eat the meat. Additional insights come from a study by Bray et al. (2016), who investigated parents' conversations with their children about the origins of meat. Women in the study were more likely than men to state that they would have preferred to avoid these conversations with their children, and they felt more conflicted about eating meat themselves.

Convergent evidence comes from Kupsala (2018) who also identified some gender differences in her qualitative material. The study included five focus groups of gastronomes, hunters, organic consumers, rural women and supermarket customers. Female-only groups (i.e., organic consumers and rural women) and groups with a majority of women (i.e., supermarket costumers) expressed more tension related to the thought of killing animals for food than groups with a majority of men. For instance, the supermarket customer group preferred products that did not remind them of animal origins, and showed a strong motivation to avoid cues that highlighted the meat-animal connection. Affective responses were strong among women who had personally been in contact with animals in food production, resulting in a clear reminder of the meat-animal connection. Moreover, the primarily female informants in the organic consumer and supermarket customer groups reported that contact with, and personification of, food producing animals would sometimes make it impossible for them to eat animal products. However, despite this evidence for dissociation possibly playing more of a role among women than men, it is important to note that rural women also criticized the de-animalization of products. More generally, when interpreting the potential gender differences in Kupsala's (2018) study, it is important to note that both the organic consumer group and the supermarket group were from urban areas, and the two groups also had a lower average age than the other groups. Hence, gender differences may have been confounded by other demographic characteristics.

In line with these qualitative findings, in the correlational study by Rothgerber (2013), women relied more on "indirect strategies" to justify their meat consumption, such as dissociation, whereas men relied more on "direct strategies," such as hierarchical, fate, and pro-meat justifications. Interestingly, while gender differences in more direct strategies were substantially reduced when controlling for masculine traits (e.g., toughness), gender differences in dissociation were relatively robust to this control. Consistent with these findings, Dowsett et al. (2018) found that women in particular showed decreased meat attachment, increased negative affect, and greater concern for animal welfare when the meat-animal link was made salient. Moreover, Kubberød et al. (2008) presented some evidence that women but not men showed increased levels of disgust in conditions involving high meat typicality (using pictures of red meat) and personification of meat (by adding a pet name related to the meat in question).

Despite these findings, it should also be noted that several studies have failed to find significant gender differences. No significant gender interactions were found in the dissociation study of Piazza et al. (2018), although exploratory post-hoc analyses suggested that dissociation tended to reduce appetite in particular among women when a baby rather than adult animal was presented. Neither, Kunst and Hohle (2016), Zickfeld et al. (2018) or Lewis (2018) found any significant gender moderation of the effects of dissociation on willingness to eat meat or on empathy and disgust. Hence, evidence on the role that gender plays for dissociation seems to be mixed. Some studies suggest that women rely more actively on dissociation than men, and that women's attitudes are particularly affected when the dissociation process is interrupted or made difficult. Yet, other studies, most of which were conducted in the U.S., suggest an absence of gender differences.
Future research is hence needed to further explore the influence of gender. Such research should also test whether the effects of gender interact with the cultural contexts the studies were conducted in. For instance, traditional male and female responsibilities in the household (and in relation to meat and food preparation) may vary from culture to culture (see, for instance, Van De Vijver, 2007) and this might lead to gender differences in dissociation in some cultures but not others. For instance, especially in less industrialized societies, the gender that traditionally has most contact with domesticated animals and their processing for meat may be less affected by dissociation. Future research may also investigate to which extent gender differences may be explained by differences in disgust sensitivity and trait empathic concern. Previous research shows that women quite consistently show more disgust sensitivity (Haidt, McCauley, \& Rozin, 1994) and to some extent empathic concern, although evidence for the latter has been less consistent (see Lennon \& Eisenberg, 1987, for a review). Hence, it is possible that whether or not men and women in a culture show differences on these traits determines whether gender differences in dissociation can be observed.

\subsection{Age differences}

Given the increased separation of meat production and consumption in many societies during the last decades, another question involves the role of age or generational (cohort) differences in the experiences of dissociation. Kupsala (2018) found differences between the groups with the highest and lowest average age. The youngest group, which consisted of supermarket costumers, expressed the least awareness of the meat-animal link, and the highest need and motivation to dissociate. By contrast, older participants would often mention and criticize "people nowadays" and how meat at present-day would be sold without head or bones. However, as mentioned before, because the interviewed groups differed in several demographic characteristics, these differences cannot conclusively be attributed to age or generation.

Several studies identified in this review used samples of adolescents or young adults. Kubberød et al. (2002) interviewed high school students and found that they had a very narrow view of what meat is. Processed and white meat was not even thought of as "meat," and the participants had difficulties verbalizing the origin of different meats without being prompted. This might indicate a high degree of dissociation among young people, particularly in the case of meat that lacks reminders of animal origins. In her master's thesis, Holm (2018) explored meat-induced cognitive dissonance and how young Norwegian adults dealt with it. Thoughts about the animal-meat connection resulted in cognitive dissonance for many of the informants, even among a few who reported little concern about animal welfare. Holm (2018) found that it often took the informants several years to gain awareness of the animal-meat connection, highlighting a potential developmental trajectory. The young informants generally preferred highly processed meat and expressed disgust and sadness when dissociation was difficult. Similarly, Kubberød et al. (2008) found that disgust for red meat was significantly higher in their sample of high school students than in their adult sample.

Despite these suggestive findings, caution should be exercised in interpreting the role of age in the studies assessed in this review. Most of the studies used samples of relatively young adults, and only few included different age groups for comparison. Studies that included older adults, such as Kubberød et al. (2008), did not make more nuanced distinctions between age groups within their adult sample. The state of evidence, hence, makes it difficult to draw clear conclusions regarding age differences in dissociation, although tentative evidence suggests that younger individuals use dissociation more and are more affected by interruption of it than older individuals. However, the existing research makes it difficult to distinguish between age and generational or cohort effects that may be overlapping or have unique influences. Further research is thus needed to understand potential 
developmental trajectories of dissociation and the role of experiences shared by age cohorts.

\subsection{Differences between rural and urban populations}

Building on the process of hedonic adaptation (Rozin, 2008), being regularly exposed to or involved in the food production process may make individuals less sensitive to reminders of meat's animal origins. As such, rural consumers who typically live closer to farms and food production facilities may be less affected by interruptions of the dissociation process and, thus, have less of a need to use it as a strategy to reduce discomfort. In support of this, Bray et al. (2016) found that urban parents were less comfortable than rural parents talking about meat production with their children. Rural parents had little problems talking about the issue because farming realities and food production were more familiar and commonplace for them. Kupsala (2018) included a focus group with rural women that was compared to an urban supermarket customer group in which all but one participant had little contact with the meat production process. The informants in the different groups talked very differently about preferences for meat. The supermarket customers, on the one hand, expressed that they preferred meat products that did not remind them of the animal-meat link, hence, enabling them to dissociate. Furthermore, they reported dislike of visible blood in meat because it evoked the idea of a once living animal. The group of rural women, on the other hand, complained about the low availability of non-processed meats. They also criticized not using all parts of the animal and did not have any problems with meats that visibly resembled their animal origins. These results seem suggestive of differences between rural and urban populations, but as stated earlier, due to the design of the study, findings cannot be conclusively attributed to specific demographic differences.

Kubberød et al. (2002) explicitly compared groups from rural and urban areas but found little evidence for differences between these groups. However, respondents who had regular contact with farm animals had a less tense relationship with animal production and meat eating. This indicates that simply living in a rural area does not necessarily affect dissociation, but that an often higher involvement in the food production might. Schröder and McEachern (2004) also found no pronounced differences between their rural and urban informants. Rural consumers were more likely to buy locally-farmed meat, however, neither urban nor rural respondents exhibited much knowledge about meat production systems or an interest in consuming "consciously." Moreover, both urban and rural consumers in the study agreed that meat packaging and presentation functioned to conceal the link between the meat and the once living animal. Both groups of respondents also stated that if pictures of tied up pigs or pigs in stalls would be presented on packaging of pork meat, or pictures of caged hens on egg cartons, they would not purchase the product in question.

Overall, the findings on differences between rural and urban populations are inconclusive, and existing evidence seems circumstantial. One interesting avenue for future research may be to longitudinally compare the dissociation tendency among people moving from rural to urban areas and vice versa. Moreover, future research should directly compare urban and rural populations but take a nuanced approach to rural populations that often are very heterogeneous. Living in a rural area may play less of a role for consumers who still buy their meat in supermarkets and keep a distance to domesticated animals than for those who work in the farming industry for instance.

\subsection{Cross-cultural variations}

A clear limitation of the currently available research on dissociation is that the majority of studies were conducted in industrialized, Western nations. This focus limits the generalizability of findings and the extent to which dissociation can be regarded as a universal phenomenon (see Henrich, Heine, \& Norenzayan, 2010). Processed meats and meats without strong cues of their animal origins are most common in Western supermarkets. By contrast, it is often more common to have whole animal carcasses on display at markets and restaurants in non-Western societies, although many exceptions exist. Additionally, in some of these societies, the slaughtering of animals for the production of meat is sometimes performed live at local markets or by families themselves, which can be regarded as a ritualization of the process (see Bastian \& Loughnan, 2017). Following the principle of hedonic adaptation, these differences may arguably make non-Westerners less sensitive to cues of the animal-meat link than Westerners.

Only three studies in this review included participants from nonWestern countries. Rothgerber (2014) included some Indian participants with their percentage ranging from $1 \%$ to $19 \%$ across five studies. Because of the small sample size, none of the effects for the Indian sample were statistically significant. Rothgerber (2014) deemed the findings in the Indian sample "tentative and unreliable," and based his discussion on the sample information and results of the participants from the United States.

The two remaining studies with non-western participants were cross-cultural studies (Kunst \& Haugestad, 2018; Tian et al., 2016). Kunst and Haugestad (2018) found that interrupting the dissociation process increased disgust and empathy both among participants from the United States and Ecuador, but willingness to eat meat was causally affected only in the United States. Importantly, these cultural differences were explained by differences in participants' exposure to unprocessed meat, highlighting the role of contextual factors. In one study, Tian et al. (2016) found that both Chinese and French participants reported less willingness to eat meat when dissociation was made difficult, but that French participants in particular engaged in additional strategies to reduce their discomfort such as denying mind to animals. However, in another study, no such differences were observed.

Evans and Miele (2012) used qualitative data from focus groups with participants from France, Italy, Norway, Sweden, Hungary, the U.K. and the Netherlands. A report by the International Monetary Fund classifies Hungary as a part of "emerging and developing Europe" as compared to the other countries in this report that are seen as more highly developed (International Monetary Fund, 2018). Additionally, Hungary has more local food supply chains compared to countries such as the Netherlands and the U.K. (Evans \& Miele, 2012). The authors found that participants from Hungary seemed to associate meat with animals to a higher degree than participants from the U.K. did. These findings indicate that national differences, and subsequent cultural and socioeconomic factors may influence the process of dissociation.

Despite tentative evidence of cultural differences, further research is needed to establish the generalizability versus culture-dependency of dissociation processes and the role of contextual moderators, such as language and social practices. For instance, research could profitably compare cultures that place different importance on meat in their diet. Moreover, languages differ in the extent to which euphemisms are employed to refer to meat products, and it would be interesting to compare whether this influences people's degree of dissociation. Finally, it may be interesting to test differences in dissociation in areas that experience rapid economic developments and consequent changes in their meat productions.

\subsection{The role of dietary patterns}

Many of the studies in this review were based on participants that consumed animal products to varying degrees. Cliceri et al. (2018) specifically analyzed how these dietary choices affected the use of dissociation. Dissociation was measured using the Meat Eating Justification scale (Rothgerber, 2013) among omnivores, vegetarians and flexitarians. Flexitarianism, or semi-vegetarianism, can be defined as actively following a meat-reduced diet without fully refraining from eating meat. Omnivores and flexitarians used dissociation equally, and significantly more so than vegetarians. Similarly, in Rothgerber (2013), 
dissociation was negatively related to different types of meat consumption and positively related to following a vegetarian diet. However, these effects fell below statistical significance, possibly due to the relatively small sample sizes of the studies. Finally, Lewis (2018) found that dissociation was moderately and positively correlated with meat consumption. This research was however part of a master's thesis and not published in a peer-reviewed journal, such that interpretations of its results must be done with caution.

In light of the available evidence, there is reason to believe that dissociation is higher among people with meat-intensive diets. Yet, further research is needed to estimate the direction of this relationship. Are people who are sensitive to disruptions of the dissociation process more likely to become vegetarians? Or does becoming vegetarian involve becoming more conscious about the animal origins of meat, thereby decreasing consumers' use of dissociation as a dissonance-reducing strategy? These are some of the questions longitudinal research may attempt to answer. There are several different motivations for following a no-meat or meat-reduced diet, such as concern about the environment, health, animal welfare or religion (Rosenfeld, 2018; Ruby, 2012). A recent review on vegetarianism by Rosenfeld (2018) suggested that vegetarians, who are motivated primarily by concerns for animal welfare, follow their diets more strictly than vegetarians with other motivations, arguably because they are more aware of the meat-animal link.

\subsection{Implications for interventions}

Finally, yet importantly, research is still needed to establish how and whether the dissociation process may be used for interventions aiming at influencing people's meat consumption. First, research assessing the effects of dissociation on behavior is urgently needed. All studies identified in this review investigated effects on self-reported meat consumption, attitudes towards meat, or intentions and willingness to eat meat. Strikingly, none of the studies included behavioral measures. Hence, to date we know very little about the extent to which the relatively robust dissociation findings observed across the reviewed studies translate into behavior. Research on justifications for eating meat have often included behavioral procedures (e.g. Bastian et al., 2012; Loughnan et al., 2010) and future research on dissociation should utilize similar designs. For instance, one simple approach may be to experimentally highlight the animal-meat link in one condition and then give participants the choice between a meat or vegetarian food product. Such a design would be a parsimonious way to test the ecological validity of the dissociation effects demonstrated in previous research and, particularly, experimental studies.

Second, further knowledge is needed about the specific aspects of thinking about animals used for meat that evoke cognitive dissonance. Is connecting meat to animals sufficient to create dissonance or is it thinking about the animal's suffering that in particular elicits it? Some evidence suggests that one reason for why priming the animal-meat connection produces cognitive dissonance is that it makes people empathic of the animals' suffering (Kunst \& Hohle, 2016). Yet, it is possible that this downstream process hinges on the animal-welfare beliefs people hold. The more consumers believe that animals suffer as part of the meat production process, the more they may potentially experience cognitive dissonance in response to animal reminders. If this is the case, interventions aiming to reduce meat consumption should be tailored to their audience. For certain populations, it may be necessary to pair reminders of the animal-meat association with information about animal suffering to create a dissonance sufficient to impact meat consumption.

\section{Conclusion}

Qualitative, correlational and experimental studies converge in support for dissociation being a process that prevents people from experiencing cognitive dissonance when consuming meat, and that consumers can use actively as a strategy to regulate the potential discomfort they may experience. The literature review indicated that dissociation is a relatively universal process, yet, that it is influenced by individual, contextual and cultural factors. Some evidence suggests that the meat consumption and affective responses of women, consumers living in industrialized Western societies, and those belonging to younger generations are particularly influenced by dissociation. Yet, evidence for these factors is inconclusive, urging the need for future research, preferably including behavioral outcomes.

\section{References}

Adams, C. J. (1990). The sexual politics of meat: A feminist-vegetarian critical theory Cambridge: Polity Press.

Bandura, A. (1999). Moral disengagement in the perpetration of inhumanities. Personality Social Psychology Review, 3(3), 193-209. https://doi.org/10.1207/ s15327957pspr0303 3.

Bastian, B., \& Loughnan, S. (2017). Resolving the meat-paradox: A motivational account of morally troublesome behavior and its maintenance. Personality and Social Psychology Review, 21(3), 278-299. https://doi.org/10.1177/1088868316647562.

Bastian, B., Loughnan, S., Haslam, N., \& Radke, H. R. M. (2012). Don't mind meat? The denial of mind to animals used for human consumption. Personality and Social Psychology Bulletin, 38(2), 247-256. https://doi.org/10.1177/0146167211424291.

Bray, H. J., Zambrano, S. C., Chur-Hansen, A., \& Ankeny, R. A. (2016). Not appropriate dinner table conversation? Talking to children about meat production. Appetite, 100, 1-9. https://doi.org/10.1016/j.appet.2016.01.029.

Cliceri, D., Spinelli, S., Dinnella, C., Prescott, J., \& Monteleone, E. (2018). The influence of psychological traits, beliefs and taste responsiveness on implicit attitudes toward plant-and animal-based dishes among vegetarians, flexitarians and omnivores. Food Quality and Preference, 68, 276-291. https://doi.org/10.1016/j.foodqual.2018.03. 020 .

Cooper, J., \& Fazio, R. H. (1984). A new look at dissonance theory. In L. Berkowitz (Vol Ed.), Advances in experimental social psychology: Vol 17, (pp. 229-266). Academic Press.

Delgado, C. L. (2003). Rising consumption of meat and milk in developing countries has created a new food revolution. Journal of Nutrition, 133(11), 3907S-3910S. https:// doi.org/10.1093/jn/133.11.3907S.

Dowsett, E., Semmler, C., Bray, H., Ankeny, R. A., \& Chur-Hansen, A. (2018). Neutralising the meat paradox: Cognitive dissonance, gender, and eating animals. Appetite, 123, 280-288. https://doi.org/10.1016/j.appet.2018.01.005.

Earle, M., Hodson, G., Dhont, K., \& MacInnis, C. (2019). Eating with our eyes (closed): Effects of visually associating animals with meat on antivegan/vegetarian attitudes and meat consumption willingness. Group Processes \& Intergroup Relations, 22(6), 818-835. https://doi.org/10.1177/1368430219861848.

Evans, A. B., \& Miele, M. (2012). Between food and flesh: How animals are made to matter (and not matter) within food consumption practices. Environment and Planning D: Society and Space, 30(2), 298-314. https://doi.org/10.1068/d12810.

Festinger, L. (1957). A theory of cognitive dissonance. Evanston, Ill: Row, Peterson and Company.

Festinger, L. (1962). Cognitive dissonance. Scientific American, 207(4), 93-106 doi:stable/ 24936719.

Foer, J. S. (2009). Eating animals. New York: Little, Brown and Company.

Graça, J., Calheiros, M. M., \& Oliveira, A. (2014). Moral disengagement in harmful but cherished food practices? An exploration into the case of meat. Journal of Agricultural and Environmental Ethics, 27(5), 749-765. https://doi.org/10.1007/s10806-0149488-9.

Haidt, J., McCauley, C., \& Rozin, P. (1994). Individual differences in sensitivity to disgust: A scale sampling seven domains of disgust elicitors. Personality and Individual Differences, 16(5), 701-713. https://doi.org/10.1016/0191-8869(94)90212-7.

Henrich, J., Heine, S. J., \& Norenzayan, A. (2010). The weirdest people in the world? Behavioral and Brain Sciences, 33(2-3), 61-83. https://doi.org/10.1017/ S0140525X0999152X.

Holm, S. T. (2018). Is it bittersweet to eat meat? How young, single Norwegian adults feel about eating meat, and their intention to eat less. (Master). Oslo: University of Oslo.

International Monetary Fund (2018). World economic outlook: Growth slowdown. Washington DC: Precarious Recovery.

Joy, M. (2011). Why we love dogs, eat pigs, and wear cows: An introduction to carnism. San Franscisco, CA: Conari press.

Kubberød, E., Ueland, Ø., Dingstad, G. I., Risvik, E., \& Henjesand, I. J. (2008). The effect of animality in the consumption experience-a potential for disgust. Journal of Food Products Marketing, 14(3), 103-124. https://doi.org/10.1080/10454440801985985.

Kubberød, E., Ueland, Ø., Tronstad, ̊., \& Risvik, E. (2002). Attitudes towards meat and meat-eating among adolescents in Norway: A qualitative study. Appetite, 38(1), 53-62. https://doi.org/10.1006/appe. 2002.0458.

Kunst, J. R., \& Haugestad, C. A. P. (2018). The effects of dissociation on willingness to eat meat are moderated by exposure to unprocessed meat: A cross-cultural demonstration. Appetite, 120, 356-366. https://doi.org/10.1016/j.appet.2017.09.016.

Kunst, J. R., \& Hohle, S. M. (2016). Meat eaters by dissociation: How we present, prepare and talk about meat increases willingness to eat meat by reducing empathy and disgust. Appetite, 105, 758-774. https://doi.org/10.1016/j.appet.2016.07.009.

Kupsala, S. (2018). Contesting the meat-animal link and the visibility of animals killed for 
food: A focus group study in Finland. Food, Culture and Society, 21(2), 196-213. https://doi.org/10.1080/15528014.2018.1427928.

Lennon, R., \& Eisenberg, N. (1987). Gender and age differences in empathy and symathy. In N. Eisenberg, \& J. Strayer (Eds.). Empathy and its development (pp. 195-217). Cambridge: Cambridge University Press.

Leroy, F., \& Praet, I. (2015). Meat traditions. The co-evolution of humans and meat. Appetite, 90, 200-211. https://doi.org/10.1016/j.appet.2015.03.014.

Lewis, K. (2018). A meat paradox: Media's Role in Mitigating the omnivore's dilemma. (Master). The University of Southern Mississippi.

Loughnan, S., Haslam, N., \& Bastian, B. (2010). The role of meat consumption in the denial of moral status and mind to meat animals. Appetite, 55(1), 156-159. https:// doi.org/10.1016/j.appet.2010.05.043.

Morton, M. (2004). Cupboard love: A dictionary of culinary curiosities. Toronto, Canada: Insomniac Press.

Piazza, J., McLatchie, N., \& Olesen, C. (2018). Are baby animals less appetizing? Tenderness toward baby animals and appetite for meat. Anthrozoös, 31(3), 319-335. https://doi.org/10.1080/08927936.2018.1455456.

Piazza, J., Ruby, M. B., Loughnan, S., Luong, M., Kulik, J., Watkins, H. M., et al. (2015). Rationalizing meat consumption. The 4Ns. Appetite, 91, 114-128. https://doi.org/10. 1016/j.appet.2015.04.011.

Rosenfeld, D. L. (2018). The psychology of vegetarianism: Recent advances and future directions. Appetite, 131, 125-138. https://doi.org/10.1016/j.appet.2018.09.011.

Rothgerber, H. (2013). Real men don't eat (vegetable) quiche: Masculinity and the justification of meat consumption. Psychology of Men and Masculinity, 14(4), 363-375. https://doi.org/10.1037/a0030379.

Rothgerber, H. (2014). Efforts to overcome vegetarian-induced dissonance among meat eaters. Appetite, 79, 32-41. https://doi.org/10.1016/j.appet.2014.04.003.

Rothgerber, H., \& Mican, F. (2014). Childhood pet ownership, attachment to pets, and subsequent meat avoidance. The mediating role of empathy toward animals. Appetite, 79, 11-17. https://doi.org/10.1016/j.appet.2014.03.032.
Rozin, P. (2008). Hedonic "adaptation": Specific habituation to disgust/death elicitors as a result of dissecting a cadaver. Judgment and Decision Making, 3(2), 191-194 (Doi not available).

Ruby, M. B. (2012). Vegetarianism. A blossoming field of study. Appetite, 58(1), 141-150. https://doi.org/10.1016/j.appet.2011.09.019.

Schröder, M. J. A., \& McEachern, M. G. (2004). Consumer value conflicts surrounding ethical food purchase decisions: A focus on animal welfare. International Journal of Consumer Studies, 28(2), 168-177. https://doi.org/10.1111/j.1470-6431.2003. 00357.x.

Segers, Y. (2012). Food systems in the nineteenth century. In M. Bruegel (Ed.). A cultural history of food in the age of empire. London, UK: Berg Publisher.

Simons, J., Hartmann, M., Klink-Lehmann, J., Vierboom, C., \& Harlen, I. (2018). Acceptance of animal husbandry in Germany: Drivers and different ways to cope with problems. Paper presented at the international association of agricultural economists (IAAE), vancouver, British columbia.

Singer, P. (1995). Animal liberation (2 ed.). London, UK: Pimlico.

Te Velde, H., Aarts, N., \& Van Woerkum, C. (2002). Dealing with ambivalence: Farmers' and consumers' perceptions of animal welfare in livestock breeding. Journal of Agricultural and Environmental Ethics, 15(2), 203-219. https://doi.org/10.1023/ A:1015012403331.

Tian, Q., Hilton, D., \& Becker, M. (2016). Confronting the meat paradox in different cultural contexts: Reactions among Chinese and French participants. Appetite, 96, 187-194. https://doi.org/10.1016/j.appet.2015.09.009.

Van De Vijver, F. J. R. (2007). Cultural and gender differences in gender-role beliefs, sharing household task and child-care responsibilities, and well-being among immigrants and majority members in The Netherlands. Sex Roles, 57(11-12), 813-824. https://doi.org/10.1007/s11199-007-9316-z.

Zickfeld, J. H., Kunst, J. R., \& Hohle, S. M. (2018). Too sweet to eat: Exploring the effects of cuteness on meat consumption. Appetite, 120, 181-195. https://doi.org/10.1016/j. appet.2017.08.038. 